\title{
A Study of the Behavior of SE and BSE in Ultra Low Landing Voltage Condition
}

\author{
Atsushi Miyaki*, Shuichi Takeuchi*, Atsushi Muto*, Yukari Dan*, Tetsuya Sawahata*, Mine \\ Nakagawa*, Toshiharu Teranishi**, and Yutaka Majima*** \\ *Hitachi High-Technologies Corp., 11-1 Ishikawa-cho, Hitachinaka, Ibaraki, 312-0057, Japan \\ ** Department of Chemistry Grad. School of Pure and Appl. Sci. University of Tsukuba, /JST-CREST, \\ 1-1-1Tennodai, Tsukuba, Ibaraki, 305-8577 Japan , \\ *** Department of Physical Electronics, Tokyo Institute of Technology / JST-CREST, \\ 2-12-1, Ohokayama, Meguro-ku Tokyo 152-8552, Japan
}

Studies in ultra low voltage condition have been reporting the interesting phenomena such as the mismatch of the proportional relationship between the image contrast and the atomic number[1], the possibility that the information source size of BSE is shallower than that of SE[2], some of which don't follow the general logic to interpret the SEM image based on the traditional SEM principles.

In the study, we set our motivation to consider the mechanism to explain such an interesting phenomena particularly happened at the ultra low voltage situation. At first we gathered a set of SE and BSE images simultaneously at ultra low voltage condition from various kinds of specimen. Second, we compared the SE and BSE image to investigate the difference. A simulation results by CASINO [3] was also applied for the theoretical consideration. In the study the recent cold FE-SEM (Hitachi SU8000) is used. The SEM is offering the SE/BSE filtering capability even at ultra low voltage condition as shown in Fig.1.

Fig.2 shows SE and BSE imaging of the Poly Si surface at the landing voltage of 100V. In SE image, distribution of the residue is clearly confirmed as a dark colored contrast, which is accomplished by the static potential detection with high sensitivity. In BSE image, approximately 1nm height topographic information was enhanced so that the fine surface morphology both on Poly Si surface and the residue were clearly observed.

Fig.3 shows the electron scattering simulation results on Si substrate by landing voltage of 100V. BSE scattering depth was around $0.9 \mathrm{~nm}$, which imply the spatial resolution for $\mathrm{Z}$ direction, was less than $1 \mathrm{~nm}$ at $100 \mathrm{~V}$ BSE signal. In case PE scattering region is extremely shallow like as imaging by the landing voltage of $100 \mathrm{~V}$, all SE is emitted without any loss. SE will be emitted from almost the entire PE scattering region so that its information source is likely to be deeper than BSE. Nevertheless, the SE image at ultra low voltage condition has the topmost surface information due to keeping the extremely shallower PE scattering region than conventional SE imaging condition.

We also report the our examination results to acquire the spatial resolution of SE and BSE.

\section{References}

[1] Ilona Müllerová, Scanning, 23, 379, (2001)

[2] D C Joy, Electron microscopy and analysis, 175, (1987)

[3] http://www.gel.usherbrooke.ca/casino/ 


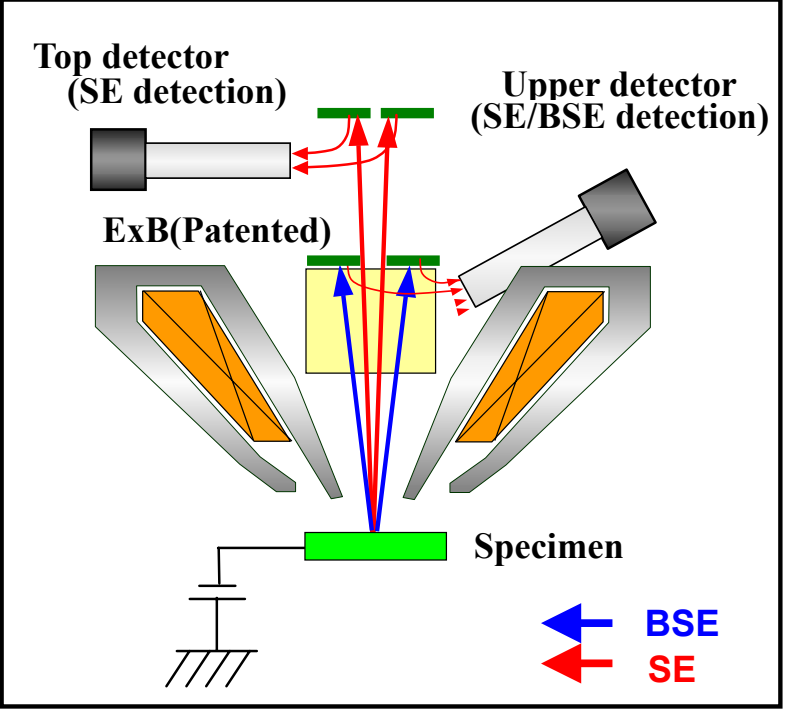

Fig.1 SE/BSE filtering function in SU8000 FE-SEM

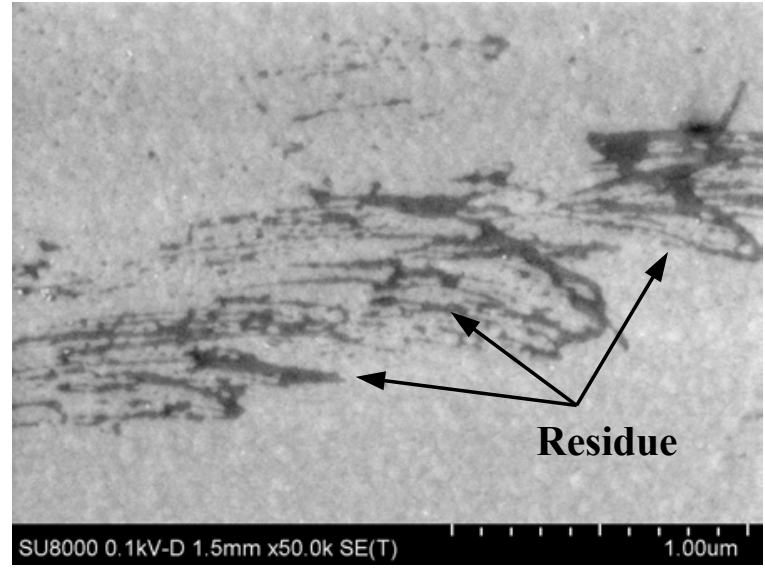

SE image

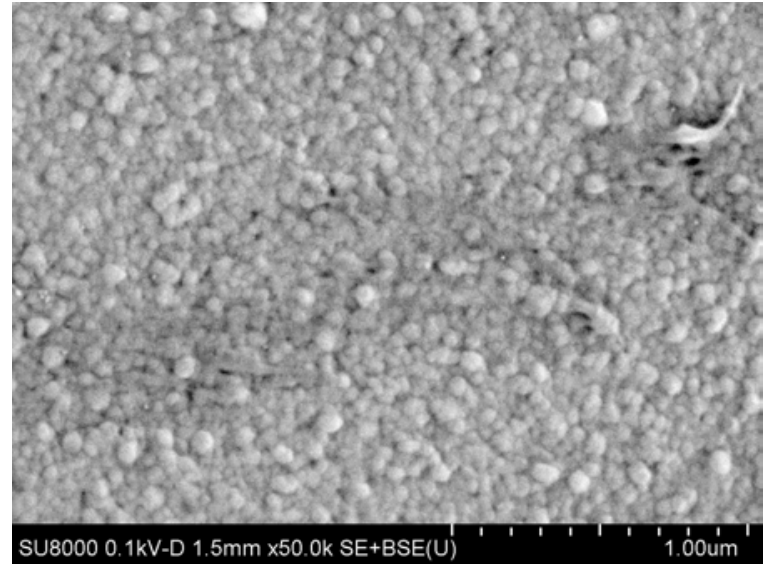

BSE image

Fig.2 SE and BSE imaging of the Poly Si surface at the landing voltage of $100 \mathrm{~V}$
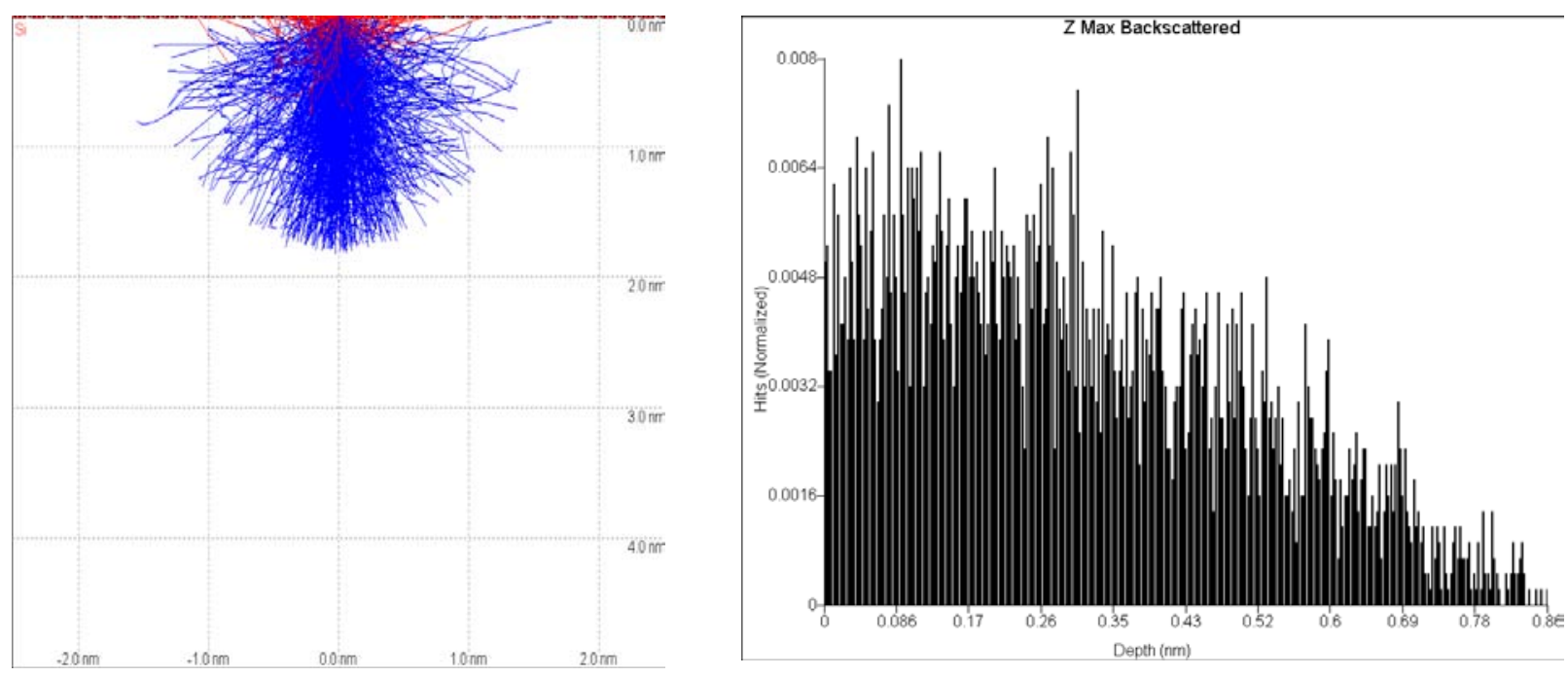

Fig.3 Electron scattering simulation results by CASINO

Substarte $\mathrm{Si}$

Landing voltage $100 \mathrm{~V}$ 